\title{
Inclined model experiment and discrete element method simulation of reinforced soil wall with leakage of backfill material
}

\author{
Takehiko Nitta ${ }^{1, *}$, Hiroaki Miyatake ${ }^{1}$, Toshikazu Sawamatsu ${ }^{1}$, Tomohiro Fujita ${ }^{1}$, Noboru Sato $^{1}$, and Masahide Otsubo ${ }^{2}$ \\ ${ }^{1}$ Public Works Research Institute, Tsukuba, Japan \\ ${ }^{2}$ The University of Tokyo, Institute of Industrial Science, Tokyo, Japan
}

\begin{abstract}
In this study, a series of inclined model experiments were conducted to investigate the behaviour of reinforced soil walls with leakage of backfill material. The experimental results were simulated by discrete element method (DEM). From the results of the inclined model experiments, it was confirmed that the tensile force in the reinforcement near the wall facing decreased due to the leakage of the backfill material, while decreasing the horizontal resistance of the reinforced soil wall remarkably. The leakage behaviour observed in the experiment was simulated using DEM. From the results of the DEM simulation, the calculated displacement pattern was found to be roughly similar to that obtained in the experiment. During leakage of the backfill material, an area without normal contact forces was generated at the lower end and this area developed toward the upper side. Such a change in normal contact forces affects the behaviour of a geogrid, in particular the disappearance of normal contact forces weakens the interlocking between the geogrid and the soil. This observation agrees with the experimental finding that the strain of the geogrid decreased in the area near the back of the wall facing.
\end{abstract}

\section{Introduction}

Owing to their outstanding stability and workability, reinforced soil walls (RSWs) have been used in many construction sites, such as roads, railways or housing lands. An efficient compaction method of backfill material is a prerequisite for the RSWs to keep their stability. In recent years in Japan, leakage of the backfill material from openings between wall facings and adjacent structure has been observed (Figure 1), especially due to large earthquakes. A significant amount of leakage may weaken the stability of the RSWs. It is necessary to evaluate the performance of the RSWs after leakage occurs so that a reliable countermeasure or repair can be proposed.

A number of research groups have studied the RSWs over decades. For earthquake condition, many shaking table experiments and inclined model experiments have been conducted to reveal the deformation behaviour of the RSWs. Inclined model experiments can apply a horizontal force to the model statically, which is the same condition as that assumed in the pseudo-static analysis used for ordinary seismic designs. According to Izawa et al. [1], RSWs subjected to seismic loading show almost the same deformation mode as those subjected to pseudo-static horizontal loading. However, in the past research, the behaviour of RSWs with leakage of backfill material has not been fully understood; thus, evaluation of the performance of them remains an open research problem. The leakage of backfill material causes flow of soil grains in the reinforced zone. Although this is a very complex

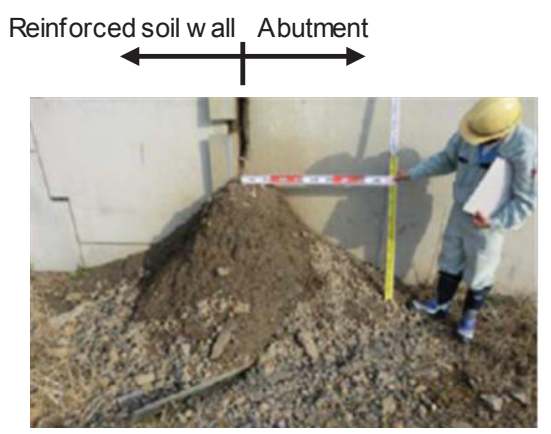

Fig. 1. Leakage of backfill material.

phenomenon, the discrete element method (DEM) enables simulations of large deformation and localization of granular material including leakage of backfill material. Prior studies have presented a geogrid modelling method and pull-out behaviour of geogrid embedded in a granular assembly [2-5]. As far as we know, however, simulations of mechanical responses of RSWs using DEM have not been reported.

This paper presents the results of verifying the performance of the RSWs with leakage of backfill material by laboratory experiments and numerical analyses. A series of inclined model experiments were conducted on the RSWs model with leakage. The movement of soil particles and the change in stress in the reinforced zone due to leakage of backfill material were simulated by DEM. Based on the soil particle behaviour, the movement of the backfill material and change in the

\footnotetext{
* Corresponding author: t-nitta44@pwri.go.jp or takehiko.nitta@terre-1.co.jp
} 
tensile force mobilised to the reinforcement were analysed.

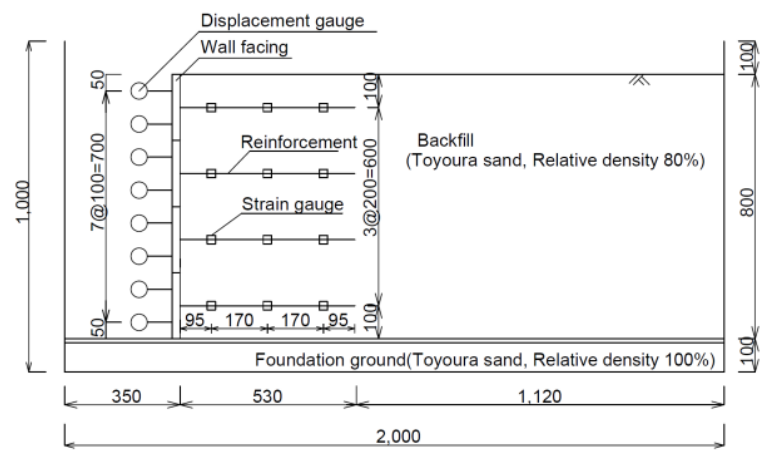

Fig. 2. Experiment model configuration (unit: $\mathrm{mm}$ ).

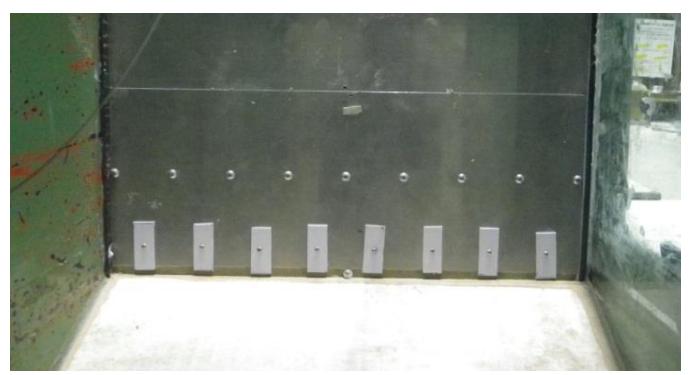

(a) Before leakage



(b) After leakage

Fig. 3. Leakage system in experiment.

\section{Inclined model experiment}

In order to reveal the influence of leakage of backfill material on the performance of the RSWs, inclined model experiments were conducted.

\subsection{Method}

\subsubsection{Model of Reinforced soil wall}

The RSWs model with a height of $800 \mathrm{~mm}$, a length of $1,650 \mathrm{~mm}$, and a depth of $500 \mathrm{~mm}$ was prepared in a soil chamber (Figure 2). Aluminium panels with a height of $200 \mathrm{~mm}$, a depth of $490 \mathrm{~mm}$ and a thickness of $6 \mathrm{~mm}$ represent the wall facings. HDPE geogrid (product standard strength was $50.0 \mathrm{kN} / \mathrm{m}$ ) was used as the geogrid models, and the wall facing and the geogrid were connected using L-shaped steel joints. The rigidity of the geogrid was reduced to about one fifth as compared with the original geogrid by thinning out the longitudinal strands. Air-dried Toyoura sand which was clean, fine and

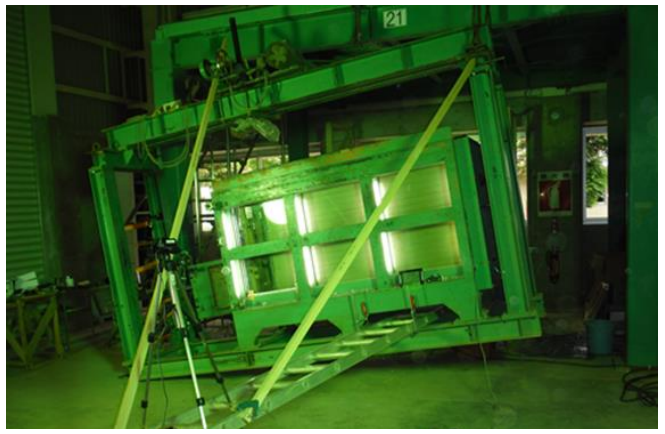

Fig. 4. Leakage system in experiment (Left: before leakage, Right: after leakage).

fairly uniform, having soil particle density of $2.64 \mathrm{~g} / \mathrm{cm}^{3}$, uniformity coefficient $D_{60} / D_{10}$ of 1.3 , mean diameter $D_{50}$ of $0.2 \mathrm{~mm}$, maximum void ratio $e_{\max }$ of 0.977 , and minimum void ratio $e_{\min }$ of 0.605 was used in all tests. The model ground was compacted to adjust the relative density to $80 \%$. The length and the spacing of the geogrid were designed based on the Japanese design and construction manual of reinforced soil using geotextile [6]. A designed safety factor $F s$ of overall stability under the horizontal seismic intensity of $k_{h}=0.4$ was 1.0 . The horizontal displacement of wall facings and the axial strain of geogrid were measured with displacement transducers and strain gauges, respectively, as schematically illustrated in Figure 2.

\subsubsection{Leakage of backfill material}

Nitta et al.[7] reported that leakage of backfill material tends to occur at openings between wall facings and adjacent structures. However, such leakage is a complicated phenomenon that behaves threedimensionally in a reinforced zone. Therefore, in order to simplify the phenomenon, in the experiment, uniform leakage of the backfill material in the depth direction of the model was artificially induced. Figure 3 shows the system of leakage of backfill material. Eight apertures with a diameter of $15 \mathrm{~mm}$ were drilled at an equal interval along the bottom wall facing. The leakage from these apertures was controlled by opening and closing valves. The amount of leaked fill material was quantified using a ratio $r_{l}$ defined as:

$$
r_{l}=W_{l} / W_{r}
$$

where $W_{r}$ is the weight of backfill material in reinforced zone, and $W_{l}$ is the weight of backfill material that leaked out.

\subsubsection{Introduction of horizontal force}

Horizontal force that represents the seismic (inertial) force due to accleration was introduced in the model by inclining the soil chamber (Figure 4). The magnitude of the horizontal force was expressed using the $k_{h}$ value in the inclined model experiment. The relationship between the $k_{h}$ value and the inclination angle of the soil chamber $\theta$ is expressed as $k_{h}=\tan \theta$. The inclination rate of the soil 
chamber was set to $1 \% \mathrm{~min}$, and the inclined state was kept for $1 \mathrm{~min}$ at every $1^{\circ}$ in order to confirm a creep deformation of model. When the inclination angle reached to a target angle, the soil chamber was returned to its original position.

\subsection{Introduction of leakage with a $r_{l}$ of $2.31 \%$}
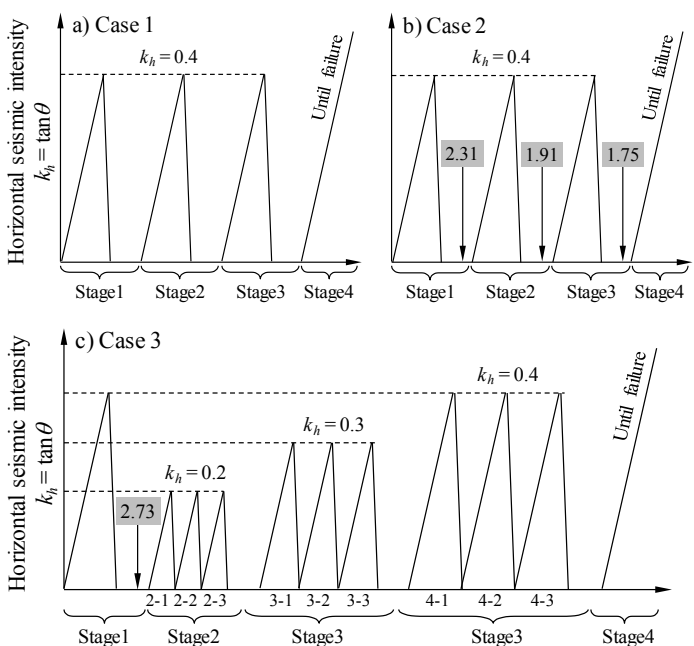

Fig.5. Experiment program.

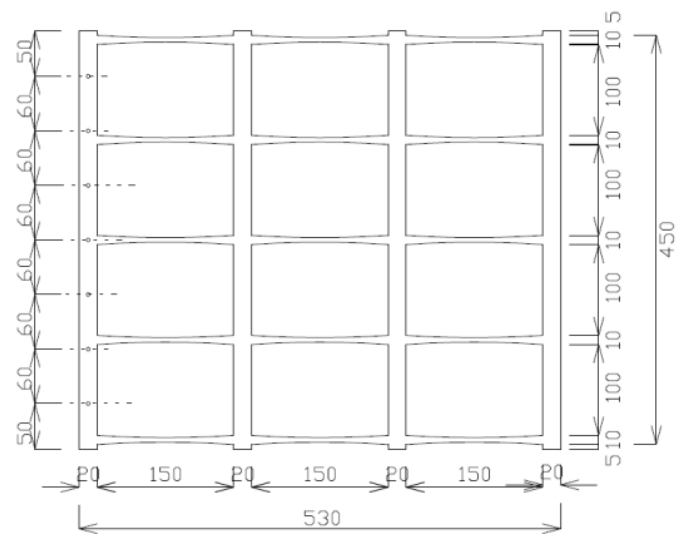

Fig. 6. Biaxial geogrid model.

\subsubsection{Experiment cases}

Referring to Figure 5 for the test programs of inclination and leakage, three test cases were conducted as below.

Case 1: No leakage was induced (Figure 5 (a)). The model was inclined to reach $k_{h}=0.4$ and returned to the original position (Stage 1), and the same procedure was repeated two more times (Stage 2 and Stage 3). After that, the model was inclined until the model failed (Stage 4). The "failure" in the experiments was defined as not only clear failure, but also when the creep displacement did not stop for 1 minute while keeping the inclination constant.

Case 2: Inclination of soil chamber and leakage of backfill material were repeated alternately (Figure 5 (b)). The inclination procedure was the same as Case 1, but the backfill material was allowed to leak at the beginning of Stage 2, Stage 3 and Stage 4. The leakage of backfill material continued until the strain of geogrid became

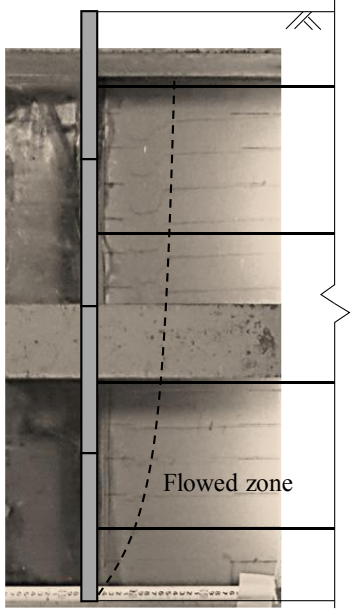

Fig. 7. Residual deformation of model ground after leakage in Case 2.

steady. As a result, the backfill material of an amount corresponding to $r_{l}$ of about $2 \%$ leaked out.

Case 3: Leakage was carried out once while repeating the inclination assuming repair of leakage (Figure 5 (c)). The leakage was introduced once after Stage 1. Peaks of inclination angle from Stage 2 to Stage 4 were gradually increased from $k_{h}=0.2$ to 0.4 and the inclination was repeated three times at each stage.

\subsection{Results}

The displacement of the wall facing, the axial strain of geogrid, ultimate horizontal capacity, as well as the soil particle behaviour due to leakage of backfill material were analysed.

\subsubsection{Behaviour of backfill during leakage}

Experimental observation on the deformation of the model ground after leakage at $r_{l}$ of $2.31 \%$ (Case 2) is illustrated in Figure 7. An area where backfill material flowed is shown by dashed line. The flowed area developed vertically from the bottom of the wall facing and reached the ground surface where a surface settlement was observed. A similar behaviour was observed in Case 3.

\subsubsection{Wall displacement and geogrid strain}

Figure 8 shows the distribution of horizontal displacement of the wall facing and geogrid strain at $k_{h}$ of 0.4 for each stage and at failure. In Case 1, the displacement of wall facing varied almost linearly along the wall height, the reinforced zone seemed to be sheared simply. The axial strain of geogrid was distributed uniformly (tensile strain) in the longitudinal direction. Although the inclination was repeated from Stage 1 to Stage 3, the displacement and the strain did not increase. In contrast, the displacement and the strain behaved characteristically due to the horizontal force applied after each process of leakage in Case 2 and Case 3. The displacement of wall facings at a higher elevation was much larger than that of Case 1, and the amount increased with repetition of leakage. However, 




(a) Case 1

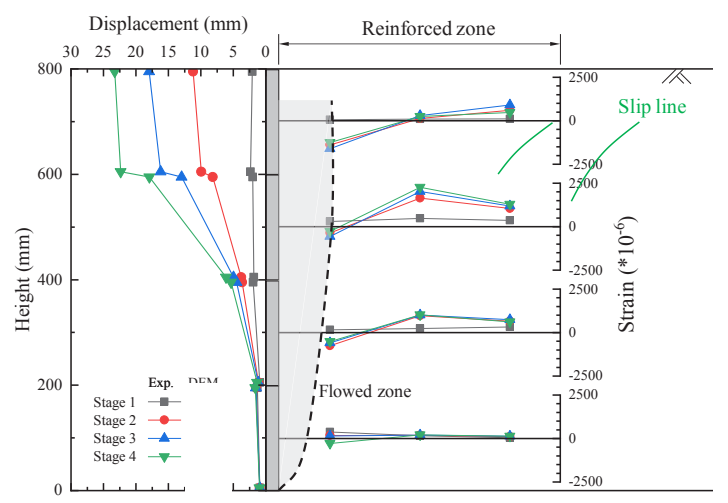

(b) Case 2

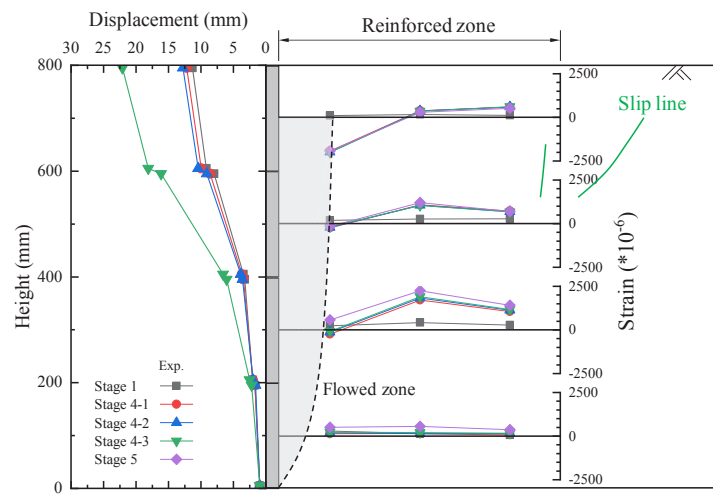

(c) Case 3

Fig. 8. Distribution of horizontal displacement of wall facing and geogrid strain at the peak inclination of each stage.

according to the result of Case 3, the displacement did not increase even when the addition of horizontal force was repeated until the model experienced the next leakage. The geogrid strain was reduced in the vicinity of the wall facing, while the strain increased in the range away from the wall facing compared to Case 1 . The range in which the amount of geogrid strain was greatly reduced corresponded to the range in which the backfill material flowed due to leakage. This indicates that the friction between geogrid and backfill material was reintroduced into the geogrid located outside the range in which flow had removed soil, and thus the geogrid strain was increased. The leakage of backfill material affected greatly to the horizontal displacement of the upper wall facing. Therefore, when evaluating the soundness of the RSWs havin kkage, the displacement of the upper wall facing should carefully observed.



Fig. 9. Horizontal seismic intensity $k_{h}$ against displacement at top wall facing.

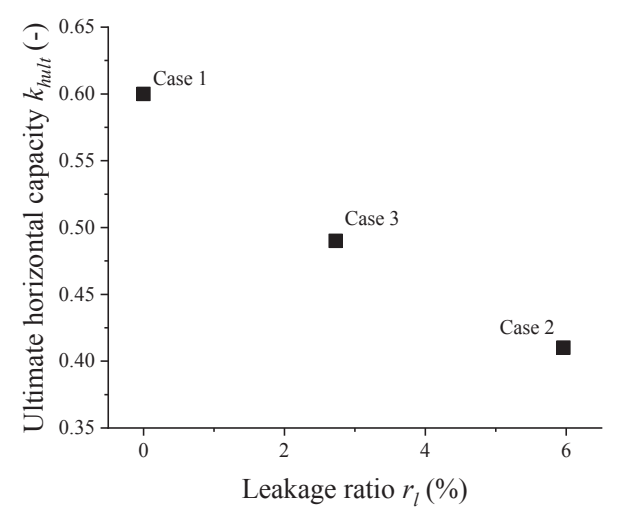

Fig. 10. Ultimate horizontal capacity $k_{\text {hult }}$ plotted against leakage ratio $r$.

\subsubsection{Horizontal force-displacement behaviour}

Figure 9 illustrates the relationship between horizontal seismic intensity and displacement of the top wall facing where the largest displacement was observed. The model responded elastically to the repeated horizontal load of $k_{h}$ $=0.4$ when no leakage was induced (Case 1). In contrast, the plastic displacement of the wall facing was confirmed due to leakage (Case 2 and Case 3), and it became significant against increase of horizontal seismic intensity after every leakage. In the relationship between force and displacement, the skeletal curve of Case 3 almost agreed with the curve of Case 2, and the response in Case 3 was elastic during the repetition of 0.2 and 0.3 of $k_{h}$. Taking these results into account, when leakage occurs due to earthquakes, it is very important to prevent promptly additional leakage in order not to enlarge the damage of RSWs due to aftershocks.

\subsubsection{Ultimate horizontal capacity}

Ultimate horizontal capacity $k_{\text {hult }}$ was defined as the horizontal seismic intensity corresponding to the failure of the model in the experiment (circle points shown in Figure 9). The $k_{\text {hult }}$ values with the leakage ratio $r_{l}$ is depicted in Figure 10. It can be clearly seen that the leakage of backfill material caused a decrease in the 
ultimate seismic intensity. The leakage ratio $r_{l}$ of Case 2 in which the leakage introduced three times was $6.0 \%$, and $r_{l}$ of Case 3 was $2.7 \%$. The $k_{\text {hult }}$ values depended on leakage ratio $r_{l} ; k_{\text {hult }}$ of Case 2 and Case 3 was $31.6 \%$ and $21.7 \%$ smaller compared to Case 1 , respectively.

\section{Numerical simulation by DEM}

In order to investigate the movement of soil particles and change in stress distribution in the reinforced zone due to leakage of backfill material, a DEM analysis was performed considering an equivalent configuration of the model tests. The numerical simulation was performed using an open-source LAMMPS software with a largescale KNL cluster system "Oakforest-PACS".

\subsection{Method}

\subsubsection{Discrete element model}

Three rigid planar surfaces were placed to simulate the side walls in the X-direction and the bottom wall of the soil chamber, and a periodic boundary was used in the Ydirection. The dimensions of the model ground were 600 $\mathrm{mm}$ in width (X-direction), $60 \mathrm{~mm}$ in depth (Y-direction) and $800 \mathrm{~mm}$ in height (Z-direction). The height of numerical model ground had the same size as that of the laboratory model ground, while the length and depth were smaller than those of the laboratory model ground. The shorter sizes were used so that the deformation characteristics of reinforced zone can be rationally analysed using a limited number of particles.

\subsubsection{Soil}

Spherical particles with a diameter of $2 \mathrm{~mm}$ were used as granular soil. A simplified Hertz-Mindlin contact model was adopted to calculate the contact response between soil particles, and between the soil particle and the rigid wall. A particle shear modulus of $29.1 \mathrm{GPa}$, Poisson's ratio of 0.23 , particle density of $2,640 \mathrm{~kg} / \mathrm{m}^{3}$ and inter-particle friction coefficient of 0.5 were used.

\subsubsection{Procedure of simulation}

Firstly, 4.4 million particles were generated randomly above the base wall having an initial void ratio $e$ about 3 , and then gravitational forces were applied to the particles with an inter-particle friction coefficient of 0.1 and a damping. Particle kinetic energy was removed by applying an additional damping after the particles settled on the base wall. The resultant e value was around 0.655 . The inter-particle friction coefficient was increased to 0.5 for the following process of simulation.

An opening was created by deleting a region of particles at the bottom end of the bottom wall facing, and the opening let soil particles leak outside the reinforced area. When soil particles corresponding to about $0.5 \%$ of $r_{l}$ leaked, the opening was closed by freezing the movement of soil particles near the opening.

\subsection{Results}

\subsubsection{Movement of soil particles}

Figures 11 and 12 are indicative of the distribution of particle displacement and particle angular velocity at leakage ratios $r_{l}$ of $0.1 \%$ and $0.5 \%$. As shown in Figure 11 , the displacement pattern is roughly similar to that obtained in the laboratory model test (Figure 7), although the area in which flow took place is wider than that obtained in the laboratory model test probably due to the difference in particle shape. The particles in the vicinity of the boundary between larger and smaller displacement (along dashed line) exhibit greater values of angular velocity (Figure 12), which corresponds closely to the boundary of particle flow. A similar distribution of angular velocity is evident between $r_{l}$ of $0.1 \%$ and $0.5 \%$, and the boundary location is unchanged, while the region of larger displacement expands upwards (Figure 11).

\subsubsection{Particle contact forces}

To discuss the particle-scale response associated with leakage, normal contact forces, connecting the centres of contacting particles were extracted from the DEM data as illustrated in Figure 13. As the contact force increases with the burial depth, the normal contact forces plotted in Figure 13 are normalised by the depth of the contact point from the ground surface. Prior to opening the bottom slit, a homogeneous distribution of normal contact forces was observed (data are not shown here). Figure 13 exhibits the normal contact forces observed at leakage ratios $r_{l}$ of $0.1 \%$ and $0.5 \%$. During leakage of the backfill material, an area without normal contact forces was generated at the lower end, and then the area expanded upwards. The direction of forces was rotated horizontally above the area without normal contact forces. This indicates development of arching. Such a change in normal contact forces affects the behaviour of a geogrid; in particular, the disappearance of normal contact forces weakens the interlocking between the geogrid and the surrounding subsoil. This observation qualitatively agrees with the experimental finding that the tensile strain of geogrid decreased in the area near the wall facing (Figure 8).

\section{Conclusions}

A series of inclined model experiments with reinforced soil wall having leakage of backfill material were performed. The results obtained have been analysed in terms of displacement of the wall facing, axial strain of reinforcement, ultimate horizontal capacity, as well as the soil particle behaviour due to leakage of backfill material. A complementary DEM simulation was conducted to understand the fundamental response of model ground associated with leakage.

The following main conclusions can be drawn from the results presented in this paper:

1) Leakage of backfill material induced the horizontal displacement of the upper wall facing due to large horizontal force. Therefore, the displacement of upper 


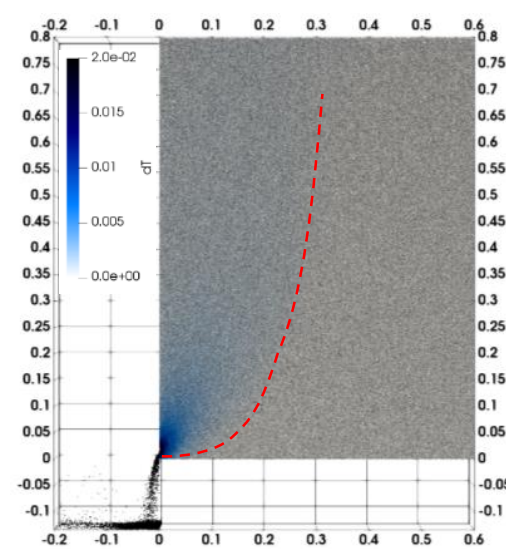

(a) $r_{l}$ of $0.1 \%$

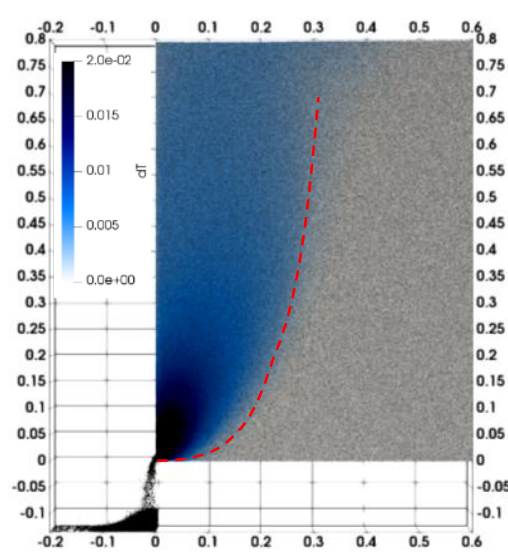

(b) $r l$ of $0.5 \%$

Fig. 11. Displacement (unit: m).

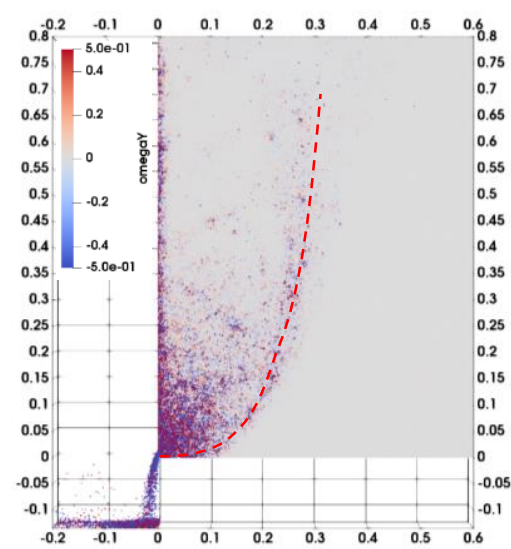

(a) $r_{l}$ of $0.1 \%$



(b) $r l$ of $0.5 \%$

Fig. 12. Angular velocity (unit: $\mathrm{rad} / \mathrm{s}$ ).

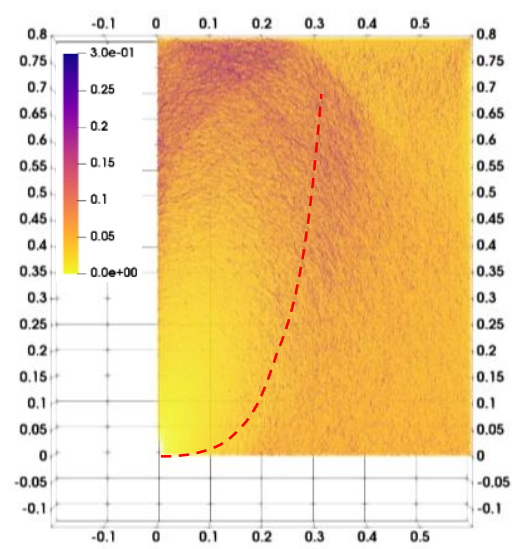

(a) $r_{l}$ of $0.1 \%$

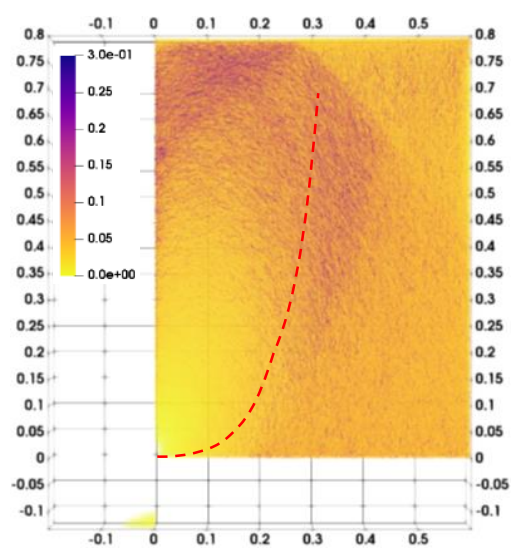

(b) $r_{l}$ of $0.5 \%$

Fig. 13. Normal contact force (unit: N). wall facing needs to be monitored carefully after earthquake to evaluate damage to RSWs having leakage of backfill material.

2) Corresponding to the area in which the soil flowed due to leakage, the geogrid strain decreased in the wall facing side and increased in the opposite side. It can be presumed that the interlocking between the soil and the geogrid was weakened inside the flowed or loosened region, while the axial force was reintroduced along the geogrid in order to balance the whole system.

3) The RSWs models having leakage behaved elastically against repeated horizontal forces until it experienced the next leakage.

4) The ultimate horizontal capacity $k_{h u l t}$ was reduced depending on the amount of leakage, i.e. reduced by about $20 \%$ and $30 \%$ at leakage ratios $r_{l}=2.7 \%$ and $6.0 \%$, respectively.

5) Leakage of backfill material can cause undesirable effects on the behaviour of RSWs. Therefore, it is necessary to take prompt measures to prevent additional leakage in order not to increase the damage of a RSWs due to aftershocks.

6) From the DEM simulation, leakage caused flow of soil particles at the back of the wall facing, and the contact force between particles decreased in the area.

7) The simulation result on particle flow revealed that the tensile strain in the geogrid can be reduced in the area near the back of the wall facing as observed in the experiment.
A part of this research was based on the Oakforest-PACS system operated at the Joint Center for Advanced HPC (JCAHPC) under cooperation with the Information Technology Center at the Univ. of Tokyo and the Center for Computational Sciences at the Univ. of Tsukuba. The modified LAMMPS code used here was developed by Dr. Kevin J. Hanley, The University of Edinburgh.

\section{References}

1. J. Izawa, J. Kuwano, Soils \& Foundations. 51 (2011)

2. J. Zhang, N. Yasufuku, H. Ochiai, Geosynthetics Eng. J. 22 (2007)

3. H. Jie, B. Anil, W. Fei, Int. J. of Geomechanics. 12 (2012)

4. V.D.H. Tran, M.A. Meguid, L.E. Chouinard, Geotextiles \& Geomembranes. 37 (2013)

5. A. Effeindzourou, B. Chareyre, K. Thoeni, A. Giacomini, F. Kneib, Geotextiles \& Geomembranes. 44 (2016)

6. PWRC, Design \& construction manual of reinforced soil using geotextile. (2013) (In Japanese)

7. T. Nitta, H. Miyatake, T. Sawamatsu, N. Sato, T. Fujita, Proc. of 7th- Chn-Jpn Geotech. Symp. (2018) 